\title{
An die Studenten
}

Wie ich bereits im Vorwort gesagt habe, war es meine Absicht, in Ihnen das Verständnis dafür zu wecken, daß die anorganische Chemie eine sich ständig erweiternde Wissenschaft ist und nicht ein abgeschlossenes Gebiet. Die meisten von Ihnen, die dieses Buch benutzen, werden bereits über Vorkenntnisse verfügen. Aus diesem Grunde habe ich mich vor allem in den letzten Kapiteln bemüht, eher einen Überblick über das Fach zu geben als ein Lehrbuch für Anfänger zu schreiben. So habe ich zum Beispiel die zitierten Arbeiten direkt in den Text einbezogen und nicht erst am Ende der einzelnen Kapitel als ,empfohlene Literatur" zusammengefaßt. Ich wollte es damit Ihnen als Lesern so leicht wie möglich machen, einen besonders interessanten Punkt zu verfolgen, und Sie dazu bringen, auch einmal die Originalliteratur zu lesen. Nach meiner Erfahrung wird die erst am Schluß eines Kapitels zitierte Literatur im allgemeinen vernachlässigt. Gegen Fußnoten wird häufig kritisch vorgebracht, daß sie den Leser ablenkten. Ich glaube nicht, daß dieser Einwand tatsächlich zutrifft. Sollte es bei Ihnen jedoch der Fall sein, wäre es gut, wenn Sie sich an diesen Stil gewöhnten. Sie werden ihm in Ihrem ganzen weiteren Berufsleben begegnen, wenn Sie wissenschaftliche Zeitschriften lesen!

Beachten Sie vor allem die kleingedruckten Abschnitte. Dort finden Sie Angaben, die für das Verstehen des behandelten Stoffes nicht notwendig sind, die jedoch interessante Nebenfragen betreffen oder die verschiedenen Seiten eines Problems weiter verfolgen.

Die Aufgaben am Schluß der einzelnen Kapitel sind ein wesentlicher Bestandteil des Buches. Einige von ihnen stellen Rückblicke auf den im Text behandelten Stoff dar, aus anderen sind Beweise für im Text Besprochenes zu entnehmen, und eine-zugegebenermaBen etwas schwierigere - Gruppe gibt Ihnen die Möglichkeit, über den Bereich des vorliegenden Buches hinausgehende Fragen zu untersuchen. Als Hilfe zur Beantwortung der Fragen enthalten viele Aufgaben Hinweise auf Besprechungen im Text oder auf die Originalliteratur. Sie sollten diese in eckigen Klammern stehenden Hinweise jedoch nicht heranziehen, ehe Sie versucht haben, die betreffende Aufgabe ohne diese Hilfe zu lösen.

Einige von Ihnen meinen sicher, ich hätte zu viel Mathematik und Quantenmechanik in die anorganische Chemie eingeführt. Denen kann ich nur den Ausspruch von Lord Kelvin entgegenhalten:

Ich betone immer wieder: Kann man das, wovon man spricht, messen und in Zahlen fassen, so weiß man etwas davon; kann man es nicht in Zahlen ausdrücken, so ist das Wissen gering und unbefriedigend - es handelt sich vielleicht um den ersten Anfang einer Kenntnis, doch ist man in seinem Denken kaum bis zur Stufe der Wissenschaft vorgedrungen, unabhängig von dem Gebiet, das es betrifft.

Andere werden dagegen der Ansicht sein, meine Erklärungen seien zu qualitativ - wie mit einer Handbewegung abgetan; ich hätte jeweils die exakte mathematische Gleichung angeben und es dabei bewenden lassen sollen. Darauf kann ich nur entgegnen, daß die Anwendung der passenden Gleichung nicht unbedingt bedeutet, daß man die physikalischen Vorgänge in dem System versteht. R. Hoffmann, einer der Erfolgreichsten in dem Bestreben, eine Synthese zwischen Theorie und Praxis zu finden, hat das so ausgèdrückt [Acc. Chem. Res. 4, 1 (1971)]:

Im Prinzip könnte man so fortfahren und jedes Molekül berechnen, ... jedoch ... selbst bei einer ausgezeichneten Übereinstimmung der Ergebnisse mit den Experimenten würde die daraus folgende 
Möglichkeit zur Voraussage nicht notwendigerweise das Verständnis einschließen. Das wirkliche Verstehen erfordert die Kenntnis der unterschiedlichen physikalischen Faktoren und des Zusammenwirkens verschiedener physikalischer Mechanismen, aus denen sich eine Größe ergibt, die man beobachten kann.

Schließlich mögen einige von Ihnen fragen: „Warum hat er so viel geschrieben?!“ Ich bitte um Verzeihung wegen der stärkeren Beanspruchung Ihres Geldbeutels und des gröBeren Gewichtes Ihrer Büchertasche, aber ich kann mich nicht entschuldigen für die Vielseitigkeit der anorganischen Chemie - im Gegenteil: Ich schwelge darin! Ich erwarte nicht, wie ich schon im Vorwort betont habe, daß in Ihrem Studium jedes Kapitel behandelt wird. Ich selbst tue es in meinen Vorlesungen auch nicht. Schließlich hat jeder von uns seine speziellen Vorlieben und Vorurteile.

Ich kenne einen Anorganiker, der Mineralien untersucht - seit alter Zeit ein Thema der anorganischen Chemie. Er tut das jedoch nicht mit einem Lötrohr und einem Stück Holzkohle, sondern versucht, mit Hilfe eines Computers mehr über die Elektronenstruktur der verschiedenen Minerale zu erfahren. Ein anderer watet hinaus ins Meer, bis zu den Knien in der Brandung - nicht gerade ein üblicher Arbeitsraum für einen Anorganiker --, um Sipunculide (Spritzwürmer) für seine Untersuchungen über Hämerythrin zu sammeln, dessen Moleküle höchstens vier Promille Eisen enthalten, der Rest ist ,organisch“ oder „biologisch“. Der dritte arbeitet in einem großen chemischen Werk an der Entwicklung besserer Katalysatoren für die petrochemische Industrie, während ein vierter geringschätzig spottet: „Das ist organische Chemie!“" Es gibt viele, mich eingeschlossen, die wissen wollen, warum cis-Diammindichloroplatin(II) in der Krebs-Therapie wirksam ist. Ein Kollege setzt vielleicht dagegen: „Platin, Ordnungszahl 78, ist 1748 von de Ulloa zum ersten Mal erwähnt worden, ein Vierteljahrhundert vor der historischen Entdeckung des Sauerstoffs durch Priestley; es bildet normalerweise Verbindungen in den Oxidationsstufen II und IV, die planar-quadratische beziehungsweise oktaedrische Struktur haben. Aber es gibt 107 andere Elemente, die alle ebenso interessant sind!" Und noch ein anderer ist fasziniert von der Tatsache, daß einige Reaktionen von Cobalt und Rhodium anscheinend der Periodizität der Eigenschaft widersprechen. Wer von ihnen hat nun Recht? Natürlich jeder! Wenn Sie dieses Buch lesen, wird, wie ich hoffe, irgendein Abschnitt, eine Einzelheit, ein Problem Ihre Phantasie entzünden, wie es bei all meinen erwähnten Freunden geschehen ist.

Und schließlich hoffe ich, daß Sie beim Lesen dieses Buches ebensoviel Freude empfinden wie ich sie beim Schreiben hatte. 